\title{
Study on Air Distribution of Displacement Ventilation in Dome Cinema in Summer
}

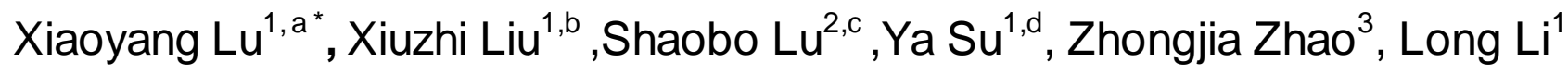 \\ ${ }^{1}$ Institute of Engineering Mechanics, Shandong Jianzhu University, Jinan 250101, China \\ ${ }^{2}$ School of Architecture, Tampere University of Technology, Tampere FI-33720, Finland \\ ${ }^{3}$ School of Civil Engineering, Shandong Jianzhu University, Jinan 250101,China \\ a luxy5504@163.com, b245443930@qq.com, '215156813@qq.com, d806032353@qq.com
}

Keywords: Dome cinema; Ground air supply; Ventilating design; Airflow organization

Abstract. This article used CFD method to simulate features of air organization in dome cinema in summer, and analyzed the temperature field, velocity field, and comfort under different designs of ventilation. The result showed that using ground air supply in large space building could be easy to satisfy the thermal comfort standard and improve air quality of workspace; rational layout of air return could accelerate airflow organization, save architecture energy and improve ventilation efficiency.

\section{Introduction}

Displacement ventilation was a new type of mechanical ventilation with air thermal buoyancy. Fresh air was sent uniformly from floor to audience zone at the state of low speed and small temperature difference, and formed strong rising currents under the joint action of indoor heat sources with heat and pollutants taken out. Compared with traditional mixing ventilation, displacement ventilation used in large space building could save energy apparently and improve air quality, thermal comfort and drafting efficiency.

\section{Airflow Organization summary in dome cinema}

Dome cinema classified as large space structure was generally used for major science and technology museum as shown in Fig. 1, cinema, and planetarium with hemispherical screen on the auditorium which had some characteristics such as high ceiling, large area, centralized staff and huge heat wet loading. And there were some demands on controlling air-conditioning noise. Hence it was very important to select the reasonable air conditioning system.

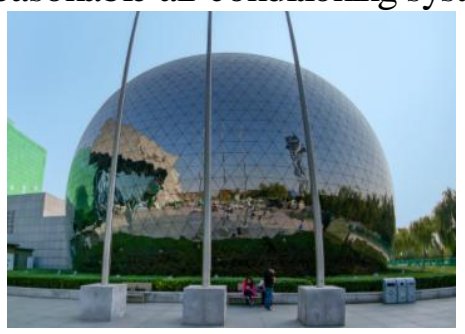

Fig.1 Outside China science and technology museum

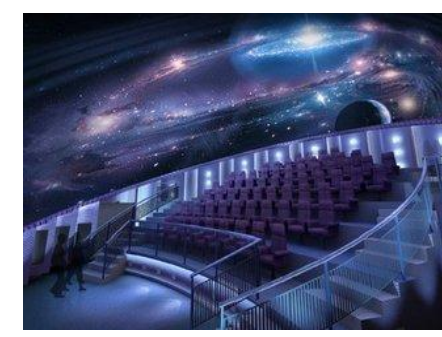

Fig.2 Arrangement of planetarium

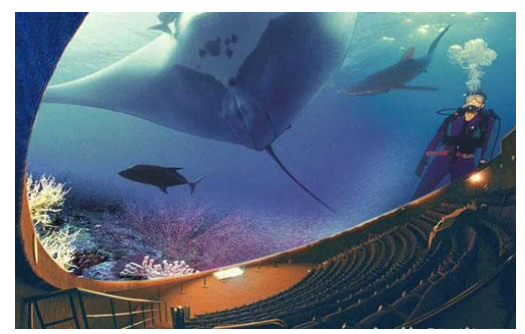

Fig.3 Arrangement of dome cinema

At present, air-conditioning ventilation system in spherical cinema mainly included displacement ventilation and hybrid ventilation in China. In order to reach the main active region, the velocity and quantity of air supply in hybrid ventilation system need to be increased, such as cosmic theatre in Jiangxi science and technology museum [1] and spherical cinema in Guangdong science center [2]. However, fresh air with low velocity and large air supply in displacement ventilation was sent directly to audience zone and took personnel heat and impure air, which benefited to save architecture energy and improve indoor air quality [3], such as planetarium in a Shanghai Museum[4] and spherical cinema in Henan Shangqiu Grand Theatre[5]. 


\section{Model simplification and construction}

The simplifying process was as followed: ignored large horizontal curvature of each step with $1.15 \mathrm{~m}$ length and $0.4 \mathrm{~m}$ width and the space occupied by seats, simplified bodies in same rank at sitting posture to entirety, and neglected heat and space of projection equipment with separate freezing and exhaust system.

\section{Physical model}

Based on the auditorium of planetarium [see Fig.2] and dome cinema [see Fig.3], three computing models containing 11 ranks and 220 seats, with a 5 meter radius and $24^{\circ}$ dips screen, were established in Fig. 4 according to different arrangements of 12 rectangular return grilles, which were set up at same distance between each other in a same ring which was in the edge of screen in model I [see Fig.4(a)], in the middle ring paralleled with the edge in model II [see Fig.4(b)], and in a horizontal ring in model III[see Fig.4(c)].

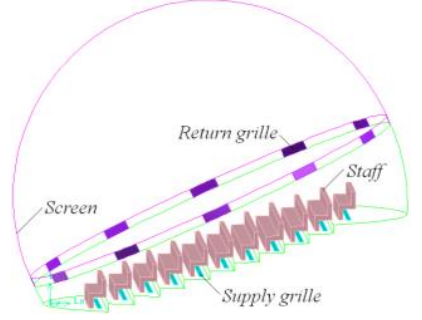

Fig.(a) model I

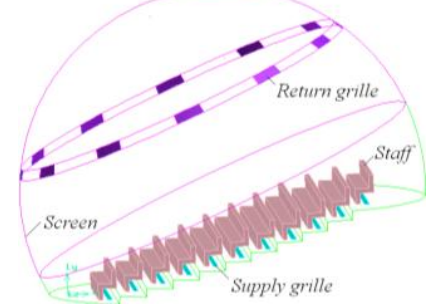

Fig.(b) model II

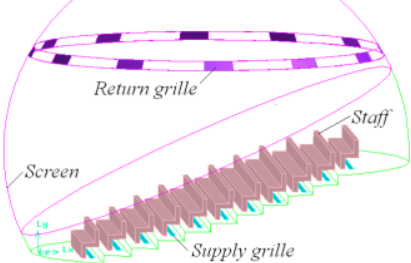

Fig.(c) model III

Fig.4 Three models of displacement ventilation

\section{Mathematical model}

Supposing that air, whose density kept with Boussinesq hypothesis, was an incompressible, radiationless, and turbulent steadily gas. Standard $k-\varepsilon$ two-equation turbulence model with wall function method and SIMPLEC algorism were applied in the calculation. The governing equations including continuity, momentum, energy, and turbulence two equation, were unified a universal transport equation[6]:

$$
\frac{\partial}{\partial t}(\rho \phi)+\frac{\partial}{\partial x_{j}}\left(\rho u_{j} \phi\right)=\frac{\partial}{\partial x_{j}}\left(\Gamma_{\phi} \frac{\partial \varphi}{\partial x_{j}}\right)+S_{\phi}
$$

where $\phi=u(j=1,2,3)$ represented the velocity of $u, v, w$ respectively, $\phi=k$ was turbulent kinetic energy, $\phi=\varepsilon$ was turbulent energy dissipating rate, $\phi=1$ was continuity equation, $x_{j}$ was coordinate, $\Gamma_{\phi}$ was generalized diffusion coefficient, and $S_{\phi}$ was generalized source.

\section{Boundary conditions and meshing}

The design temperature of auditorium in summer was $24^{\circ} \mathrm{C}$. The body temperature was $37^{\circ} \mathrm{C}$ with heat flux $50 \mathrm{~W} / \mathrm{m}^{2}$. Heat flux of screen was $10 \mathrm{~W} / \mathrm{m}^{2}$. The floor and wall around was isothermal and adiabatic. Air supply grille with $0.55 \mathrm{~m} \times 0.20 \mathrm{~m}$ size was velocity inlet boundary condition with temperature $22^{\circ} \mathrm{C}$ and velocity $0.20 \mathrm{~m} / \mathrm{s}$. Air return grille with $1.25 \mathrm{~m} \times 0.40 \mathrm{~m}$ size was outflow boundary condition. Each model was meshed fractionally into mixture grids about 700000 .

\section{Simulation and Results}

Because of the symmetrical model, the symmetric face $z=0$ was selected to compare the difference between three models on temperature field, velocity field and personnel comfort. 


\section{Analysis of temperature field}

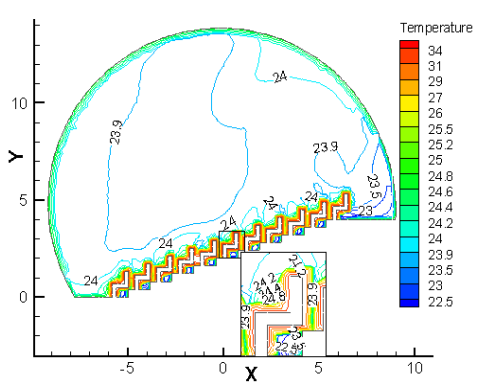

Fig.(a) model I

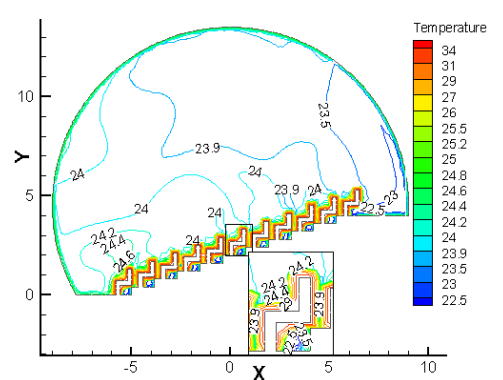

Fig.(b) model II

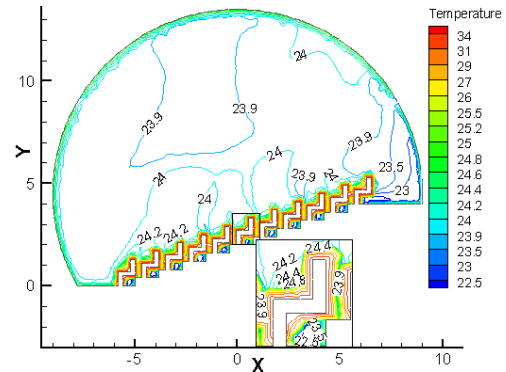

Fig.(c) model III

Fig.5 Temperature contour map of the symmetric face $\mathrm{z}=0$ in 3 models

As shown in Fig.5, temperature field of whole space had no blind corner and balanced comparatively at about $24^{\circ} \mathrm{C}$ which basically equaled to design temperature. The temperature gradient near body was relatively larger than upper space, and temperature between ranks was uniform about $23.9^{\circ} \mathrm{C}$.

Arrangement of return grille influenced the temperature field slightly. Temperature value above head kept about $24^{\circ} \mathrm{C}$ basically in model I , while $24.2^{\circ} \mathrm{C}$ in model II and III. Temperature over the front two rows in model II [see Fig.5(b)] was about $0.4^{\circ} \mathrm{C}$ much higher than that of the other two models, and temperature difference on screen also existed because of the incline ring of return grille and the distance between supply and return grille.

\section{Analysis of velocity field}

As shown in Fig.6, velocity value in whole space emerged the stratification phenomenon that velocity of upper space was about $0.05 \mathrm{~m} / \mathrm{s}$, while lower was about $0.02 \mathrm{~m} / \mathrm{s}$. That velocity isoline near outlet was more intensive meant velocity gradient was bigger. Velocity of fresh air was $0.02 \mathrm{~m} / \mathrm{s}$ when it reached body, as a result of flowing slowly after pouring. And maximum velocity between ranks was about $0.1 \mathrm{~m} / \mathrm{s}$ due to superposition.

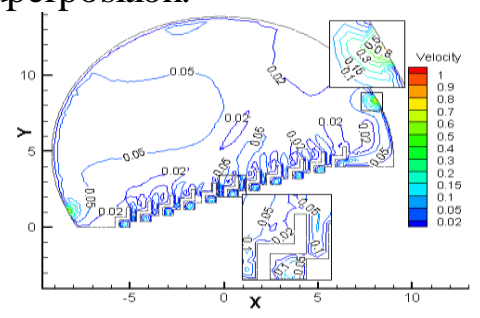

Fig. (a) model I

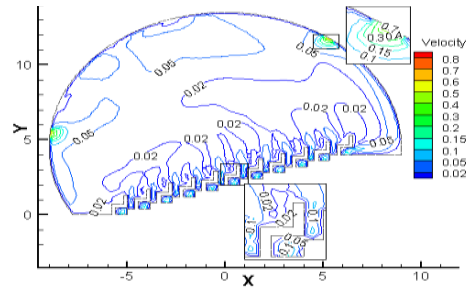

Fig. (b) model II

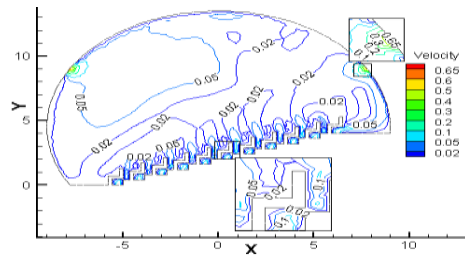

Fig. (c) modelIII

Fig. 6 velocity contour map of the symmetric face $\mathrm{z}=0$ in 3 models

Arrangement of return grille was of certain influence to the velocity field. Maximum velocity around outlet in model I [see Fig.6(a)] was $1.1 \mathrm{~m} / \mathrm{s}$, and region in which velocity was slightly bigger than $0.05 \mathrm{~m} / \mathrm{s}$ was wider than that of the other two models owing to smaller distance between inlet and outlet, while smaller velocity of upper zone benefited to control noise. Combination of outlet and supply direction [see Fig.6(c)] could promote air circuit and reduce air age.

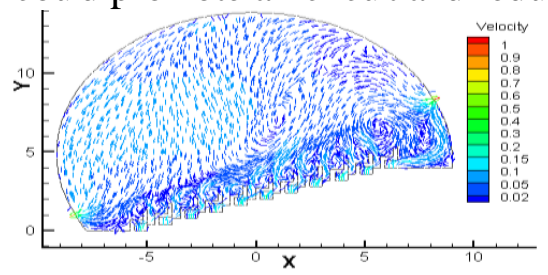

Fig. (a) model I

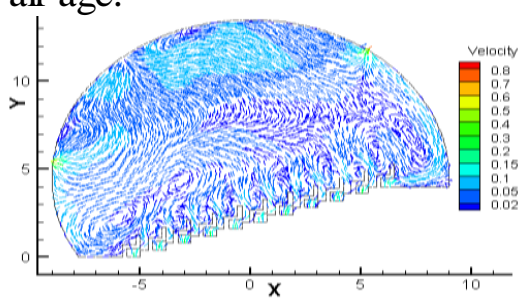

Fig. (b) model II

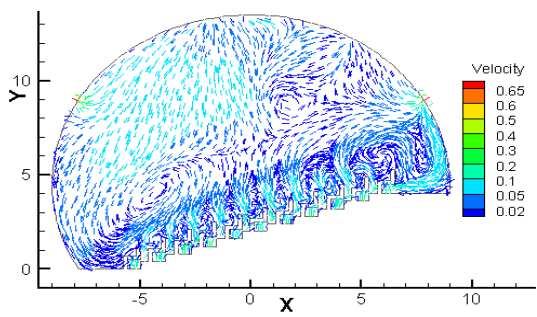

Fig. (c) modelIII

Fig.7 Velocity vector map of the symmetric face $\mathrm{z}=0$ in 3 models

(Color presented the value of velocity, arrow presented direction.) 
Body, stage and other obstacles could change original direction of fresh air which took heat and pollution away from body surrounding after entering workspace, and participated in vortex to push the air movement when it poured out and met the backing current from screen. The surrounding area was so near to outlet that air displaced rapidly, while middle air flowed out after vortex. The declining plane of outlet [see Fig.7(b)] would make the air flow to the lower return grille because of the large distance from supply to return grille, and vortex was seen obviously in modelIII[see Fig.7(c)] since the plane was perpendicular to the direction of fresh air according to closest distance and minimum power principle.

\section{Comfort analysis}

Based on summer thermal comfort standard in design code of heating, ventilation and air conditioning (GB50019-2003):(a)minimum supply air temperature at $0.1 \mathrm{~m}$ away from floor $t_{0.1 \mathrm{~min}} \geq 21^{\circ} \mathrm{C}$;(b)vertical temperature difference between head and foot $\Delta t_{0.1 \sim 1.1} \leq 2{ }^{\circ} \mathrm{C}$ and $\Delta t_{0.1 \sim 1.8} \leq 3{ }^{\circ} \mathrm{C}$; (c)maximum velocity value in workspace $V_{0.1} \leq 0.25 \mathrm{~m} / \mathrm{s}$. Comparison the above parameters at different locations showed that ground air supply could meet the above standard.

\section{Conclusion}

Through simulation and analysis on temperature and velocity field in dome cinema auditorium under different arrangements of displacement ventilation, the results showed that:

(1) Three models could satisfy the comfort standard on temperature, vertical temperature difference and wind velocity. Ground air supply was a comparative energy-saving mode and easy to reach the comfort standard of workspace.

(2) Although the layout of return air grille could exert a measure of influence over the temperature, velocity and airflow within auditorium, the comfort of workspace surrounding staff was mainly determined by the arrangement of ground air supply inlet.

(3) Increasing distance from inlet to outlet appropriately could promote upper airflow and improve ventilation efficiency, and also could cut down the air age and give the screen cooling effect.

\section{Acknowledgements}

This project was financially supported by the Graduate Student Innovation Plan of Shandong Province (SDYY08038).

\section{References}

[1] Chunlin Shu, Heat and ventilating design of Cosmic Theatre in Jiangxi science and technology Museum, J. Refrigeration Air Conditioning \& Electric Power Machinery. 91.24 (2003) 35-36,40 In Chinese

[2] Chunxiang Wang, Youcai Ma, Huabin Liu, Air conditioning and ventilating design of science and technology cinemas, J. HV\&AC. 39.3 (2009) 24-26 In Chinese

[3] Xiaoyang Lu, Ya Su, Shaobo Lu, Numerical Analysis on Stratified Air Conditioning of Air Distribution in a Auditorium/Hall, The fourth International Conference on Energy and Environmrntal Protection, (2015)

[4] Jialong Ren, Design of Air-conditioning System for the Planetarium, J. Refrigeration Air Conditioning \& Electric Power Machinery. 139. 32 (2011) 36-39 In Chinese

[5] Jie Liu, Air Conditioning Design for Grand Theatre of Shangqiu, J. Building Energy Efficiency.43 290 (2015) 29-33 In Chinese

[6] Patankar SV. Numerical Heat Transfer and Fluid Flow, Mc-Graw-Hill, New York, 1980. 